Journal Club

Editor's Note: These short, critical reviews of recent papers in the Journal, written exclusively by graduate students or postdoctoral fellows, are intended to summarize the important findings of the paper and provide additional insight and commentary. For more information on the format and purpose of the Journal Club, please see http://www.jneurosci.org/misc/ifa_features.shtml.

\title{
Phosphorylation of AMPA-Type Glutamate Receptors: The Trigger of Epileptogenesis?
}

\author{
Joanne Bakker, ${ }^{\star}$ Frederike J. Basedow, ${ }^{\star}$ Alain D. Dekker, ${ }^{\star}$ and Christos Papantoniou ${ }^{\star}$ \\ Department of Molecular Neurobiology, Graduate School of Behavioural and Cognitive Neurosciences, University of Groningen and University Medical \\ Center Groningen, Nijenborgh 7, 9747 AG Groningen, The Netherlands \\ Review of Rakhade et al.
}

Epilepsy is a common neurological disease characterized by changes in consciousness, recurrent unprovoked seizures, and signs of abnormal neurological activity. Epilepsy, present in $\sim 40$ different types, affects 50-65 million people in the world, mostly young children and people older than 65. Infants who experience neonatal seizures have a higher incidence of developing epilepsy in later life. Moreover, seizures are associated with later-life psychiatric and cognitive dysfunction (Rakhade et al. 2012). The underlying mechanisms of epilepsy have still not been discovered, limiting its treatment possibilities. Although drugs that reduce the frequency and severity of seizures are currently available, no treatment has yet been developed that prevents seizures.

A potentially promising therapeutic target to prevent epileptogenesis is the AMPAtype glutamate receptor (AMPAR). In a rat model of neonatal seizures, intraperitoneal administration of AMPAR antagonists within $48 \mathrm{~h}$ of early-life seizures reduced later-life seizure susceptibility and hippocampal neural injury (for review, see Rakhade et al. 2008). Furthermore, hypoxia-induced seizures altered AMPAR

Received Feb. 1, 2013; revised Feb. 27, 2013; accepted March 1, 2013.

We thank Ulrich L.M. Eisel, professor in Molecular Neurobiology (University of Groningen), for his useful advice.

*All authors contributed equally to this review.

Correspondence should be addressed to Christos Papantoniou, Ni-

jenborgh 7, 9747 AG Groningen, The Netherlands. E-mail: chr6979@gmail.com.

DOI:10.1523/JNEUROSCI.0496-13.2013

Copyright $\odot 2013$ the authors $\quad 0270-6474 / 13 / 335879-02 \$ 15.00 / 0$ signaling in immature rats by rapidly increasing the amplitude and frequency of AMPAR-mediated synaptic currents (Rakhade et al. 2008). Administration of AMPAR antagonists after seizures reduced kinase activity and phosphorylation of AMPAR subunits, thereby preventing an increase in seizure susceptibility (Rakhade et al. 2008). Therefore, changes in AMPAR phosphorylation seem important for epileptogenesis.

AMPARs are ligand-gated ion channels that consist of combinations of four different subunits, GluR1-4. Their levels at the synaptic membrane are dynamic, and strongly dependent upon phosphorylation of AMPAR serine sites. For example, phosphorylation of GluR1 at S845 increases cell surface and synaptic AMPAR levels (He et al. 2011), thus increasing excitability. Furthermore, phosphorylation of S831 and S845 of GluR1 contributes to long-term potentiation (LTP) by increasing AMPAR conductance/open channel probability. Blocking these posttranslational modifications diminishes GluR1 trafficking to the membrane, thereby reducing excitability and LTP (Shepherd and Huganir, 2007). Phosphorylation of AMPAR serine sites could therefore be involved in the hyperexcitability seen in epilepsy.

To assess whether phosphorylation of GluR1 at S831 and S845 might increase seizure susceptibility, Rakhade et al. (2012) generated knock-in mice carrying mutations in S831 and S845. In these GluR1 double-phosphomutant (GluR1-DPM) mice, neither serine could be phosphorylated. The $\mathrm{GABA}_{\mathrm{A}}$ receptor antagonist Pentylenetetrazol (PTZ) was injected into mice on postnatal day 7 (P7) to induce seizures. In wild-type (WT) mice, PTZ increased GluR1 S831 and S845 phosphorylation. Although the severity of PTZ-induced seizures did not differ in WT and GluR1-DPM mice, the latency to the next seizure was delayed in GluR1-DPM mice. Moreover, a significant increase in the postsynaptic scaffolding protein PSD-95 was observed in WT, but not in GluR1-DPM mice, indicating that phosphorylation of serine sites is involved in synaptic alterations during neonatal seizures.

Similar results were found with other seizure models. Exposing WT and GluR1DPM mice to graded global hypoxia for 40 min also resulted in the development of seizures. Hypoxia-induced seizures caused phosphorylation of both GluR1 S831 and S845 in WT mice. In GluR1-DPM mice acute seizure incidence was decreased compared with WT mice. Subsequently, long-term effects on later-life seizure susceptibility were observed when seizures were induced using kainic acid (KA). WT mice with early-life hypoxia-induced seizures had a decreased latency of developing later-life KA seizures compared with naive WT mice. No difference in latency of developing later-life KA seizures was observed between GluR1-DPM mice with early-life hypoxia-induced seizures and naive GluR1DPM mice, indicating that seizure-induced phosphorylation of S831 and S845 contributes to later-life hyperexcitability and 
seizure susceptibility. Finally, human postmortem tissue was examined from cases of neonatal seizures. In this tissue, increased phosphorylation of both GluR1 S831 and S845 in the hippocampus compared with age-matched controls was found. In short, Rakhade et al. (2012) showed that phosphorylation of GluR1 at S831 and S845 might play an important role in epileptogenesis.

Phosphorylation of GluR1 might be involved in other models of epileptogenesis as well. A recent study by McMahon et al. (2012) has shown that impaired autophagy is involved in epileptogenesis. Increased activity of the mammalian target of rapamycin (mTOR) pathway decreases autophagy, and mice in which mTOR suppression is impaired display seizures, suggesting that impaired autophagy can lead to the development of seizures. Interestingly, autophagy also affects AMPAR surface expression. Shehata et al. (2012) found that inducing autophagy resulted in GluR1 degradation in hippocampal neurons, whereas decreasing autophagy restored GluR1 levels. Therefore, it can be hypothesized that hyperactivity of mTOR leads to decreased autophagy, which in turn increases AMPAR surface expression and subsequent hyperexcitability of neurons, which is the basis of seizures (Fig. 1). To our knowledge, however, no research has yet linked autophagy and GluR1 phosphorylation; impaired autophagy has only been shown to be involved in the degradation of this receptor subtype.

Another factor that has been shown to be involved in both epilepsy and AMPAR expression is inflammation. Interestingly, in a study by Galic et al. (2009), viral-like brain inflammation starting at P14 increased expression of GluR1 mRNA in the hippocampus and increased seizure susceptibility in adult rats. This effect of inflammation on GluR1 expression might be mediated by tumor necrosis factor $\alpha$ (TNF- $\alpha$ ), a proinflammatory cytokine that increases insertion of AMPARs in the plasma membrane (Shepherd and Huganir, 2007). Genetic deletion of TNF Receptor 1 (TNFR1) suppressed excitatory synaptic transmission by decreasing the surface expression and synaptic localization of GluR1 (He et al., 2012). Thus, inflammation, potentially via TNF- $\alpha$, increases GluR1 surface expression, which may enhance the devel-

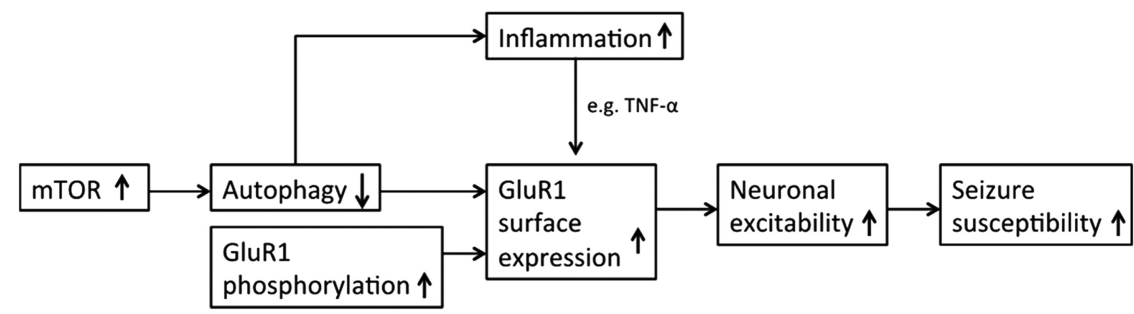

Figure 1. Surface expression of GluR1 might constitute a link between autophagy, inflammation, and epilepsy. Evidence from studies supporting these links is described in the text.

opment of seizures. As with autophagy, however, whether the observed elevation of GluR1 surface expression in response to inflammation results from increased GluR1 phosphorylation remains unknown.

The possible involvement of impaired autophagy and inflammation in epileptogenesis raises the question of whether these processes play a collaborating role in the development of seizures. The connections between autophagy and inflammation are complex. Autophagy is important in the regulation of inflammation and the coordination of innate and adaptive immune responses (Jo et al., 2012). Cellular stressors might activate autophagy, which could decrease inflammation and prevent inflammatory injury. Defects in the regulation of autophagy have been shown to activate inflammation and lead to the development of inflammatory diseases (Jo et al., 2012); and as stated previously, McMahon et al. (2012) found a link between impaired autophagy and epilepsy. Mechanistically, impaired autophagy might enhance inflammation resulting in a higher surface expression of GluR1 and an enhanced development of seizures (Fig. 1). However, the connection between autophagy and inflammation in the development of seizures is not clear and needs further investigation.

In conclusion, the finding that phosphorylation of GluR1 S831 and S845 affect seizure susceptibility and excitability offers a new perspective on the development of therapy for epilepsy. Interestingly, AMPAR subunit expression is related to autophagy and inflammatory processes. Both have been shown to play a role in epilepsy. However, how these processes relate to AMPAR phosphorylation in the context of epilepsy is currently unclear. Future research should examine this relationship and the underlying molecular pathway. This might reveal the essential mechanisms underlying sei- zures and serve useful in discovering new therapeutic targets.

\section{References}

Galic MA, Riazi K, Henderson AK, Tsutsui S, Pittman QJ (2009) Viral-like brain inflammation during development causes increased seizure susceptibility in adult rats. Neurobiol Dis 36:343-351. CrossRef Medline

He K, Goel A, Ciarkowski CE, Song L, Lee HK (2011) Brain area specific regulation of synaptic AMPA receptors by phosphorylation. Commun Integr Biol 4:569-572. Medline

He P, Liu Q, Wu J, Shen Y (2012) Genetic deletion of TNF receptor suppresses excitatory synaptic transmission via reducing AMPA receptor synaptic localization in cortical neurons. FASEB J 26:334-345. CrossRef Medline

Jo EK, Shin DM, Choi AMK (2012) Autophagy: cellular defense to excessive inflammation. Microbes Infect 14:119-125. CrossRef Medline

McMahon J, Huang X, Yang J, Komatsu M, Yue Z, Qian J, Zhu X, Huang Y (2012) Impaired autophagy in neurons after disinhibition of mammalian target of rapamycin and its contribution to epileptogenesis. J Neurosci 32:15704-15714. CrossRef Medline

Rakhade SN, Zhou C, Aujla PK, Fishman R, Sucher NJ, Jensen FE (2008) Early alterations of AMPA receptors mediate synaptic potentiation induced by neonatal seizures. J Neurosci 28:7979-7990. CrossRef Medline

Rakhade SN, Fitzgerald EF, Klein PM, Zhou C, Sun H, Huganir RL, Jensen FE (2012) Glutamate receptor 1 phosphorylation at serine 831 and 845 modulates seizure susceptibility and hippocampal hyperexcitability after early life seizures. J Neurosci 32:17800-17812. CrossRef Medline

Shehata M, Matsumura H, Okubo-Suzuki R, Ohkawa N, Inokuchi K (2012) Neuronal stimulation induces autophagy in hippocampal neurons that is involved in AMPA receptor degradation after chemical long-term depression. J Neurosci 32:10413-10422. CrossRef Medline

Shepherd JD, Huganir RL (2007) The cell biology of synaptic plasticity: AMPA receptor trafficking. Annu Rev Cell Dev Biol 23:613-643. CrossRef Medline 